\title{
L'AFFINITÉ DE DEUX PROTOZOAIRES ÉPIBIONTES POUR LES DEUX ESPẼCES JUMELLES DE PLANAIRES D'EAU DOUCE POLYCELIS NIGRA ET POLYCELIS TENUIS
}

\author{
par C. LASCOMBE ${ }^{\mathrm{T}}$.
}

\begin{abstract}
L'étude porte sur l'association de commensalisme liant les deux Planaires jumelles Polycelis nigra et $P$. tenuis à deux Protozoaires Ciliés épibiontes de la famille des Urceolariidae. Urceolaria mitra est nettement majoritaire sur Polycelis tenuis, et Trichodina steini exclusive sur Polycelis nigra. Des expériences de choix attestent la préférence indiscutable des épibiontes pour leur espèce affine de Planaire.
\end{abstract}

\section{The affinity of two epizoic protozoa for the two sibling species of freshwater planarians Polycelis nigra and Polycelis tenuis.}

This study is concerned with the commensal relationship between two sibling Polycelis species of Triclads and two epizoic Ciliates belonging to the family Urceolariidae. Urceolaria mitra is clearly on Polycelis tenuis, and Trichodina steini exclusive on Polycelis nigra. Choice experiments demonstrated a definite preference of each epizoic Ciliate for the Triclad species on which it was found in the field.

Polycelis nigra (O. F. Muller) et Polycelis tenuis Ijima sont deux formes de Triclades paludicoles morphologiquement identiques et qui se distinguent par l'anatomie de leur appareil génital. Ces deux espèces, longtemps confondues par le passé et récemment encore étudiées globalement, n'en possèdent pas moins des caractéristiques physiologiques et écologiques propres (Reynoldson 1966, Reynoldson et Bellamy 1971, Lascombe 1971, Pattee 1972). Elles peuvent cependant coexister dans la nature.

L'observation d'un grand nombre d'individus récoltés dans la région lyonnaise a permis de constater la présence fréquente de Protozoaires fixés sur les Planaires. Ces protozoaires présentent

1. Département de Biologie Animale, Université Claude-Bernard, 43, boulevard du 11-Novembre, 69 - Villeurbanne. 
deux types morphologiques voisins; ils appartiennent à des genres différents et sont rarement portés par le même individu.

Le problème était alors de savoir si, sur le plan de leur éthologie, les deux Planaires n'apparaissaient pas également différentes et s'il ne s'établissait pas avec les Ciliés des relations spécifiques préférentielles.

Une seconde préoccupation, plus pratique, a conduit à cette étude : les problèmes posés par la détermination de $P$. nigra et $P$. tenuis. Les caractères de reconnaissance liés à la coloration (Reynoldson 1966) n'étant utilisables que pour certaines populations (Lascombe 1971), seuls les critères anatomiques (Lender 1936, Reynoldson 1958 a) restent valables pour l'identification rigoureuse des espèces. Mais ils ne sont applicables qu'à des animaux adultes, pourvus d'organes sexuels. Par ailleurs, ils nécessitent la section de l'animal et n'autorisent donc l'expérimentation que sur des animaux regénérés à partir de fragments déterminés ou nés au laboratoire de parents d'espèce connue. On perçoit les nouvelles possibilités que pourrait offrir l'apport d'un critère de détermination externe pour la reconnaissance de ces espèces.

\section{1. - DONNEES GENERALES SUR LES EPIBIONTES}

Les épibiontes étudiés sont des Ciliés Péritriches. Il s'agit de Trichodina steini Claparede et Lachmann 1857 et de Urceolaria mitra (Von Siebold) Stein 1867. Voisins des Vorticelles, ils appartiennent à la famille des Urceolariidae.

- Ils diffèrent par un certain nombre de caractères dont les plus évidents sont les suivants (fig. 1 et 2) :

\section{T. steini}

taille :

forme générale : appareil adhésif :
$40-50 \mu$

bol renversé anneau squelettique à chevrons muni de crochets très marqués.

\section{U. mitra}

$100 \mu$ bonnet phrygien anneau squelettique torsadé

Leur détermination est essentiellement basée sur ce dernier caractère (Kahl 1935).

Au cours d'une série de travaux, Reynoldson (1947 à 1956) a fourni bon nombre d'informations quant à l'écologie et la physiologie de $\boldsymbol{U}$. mitra bien que les renseignements concernant $T$. steini soient rares, il semble que sur ce plan, les deux espèces soient assez voisines. 

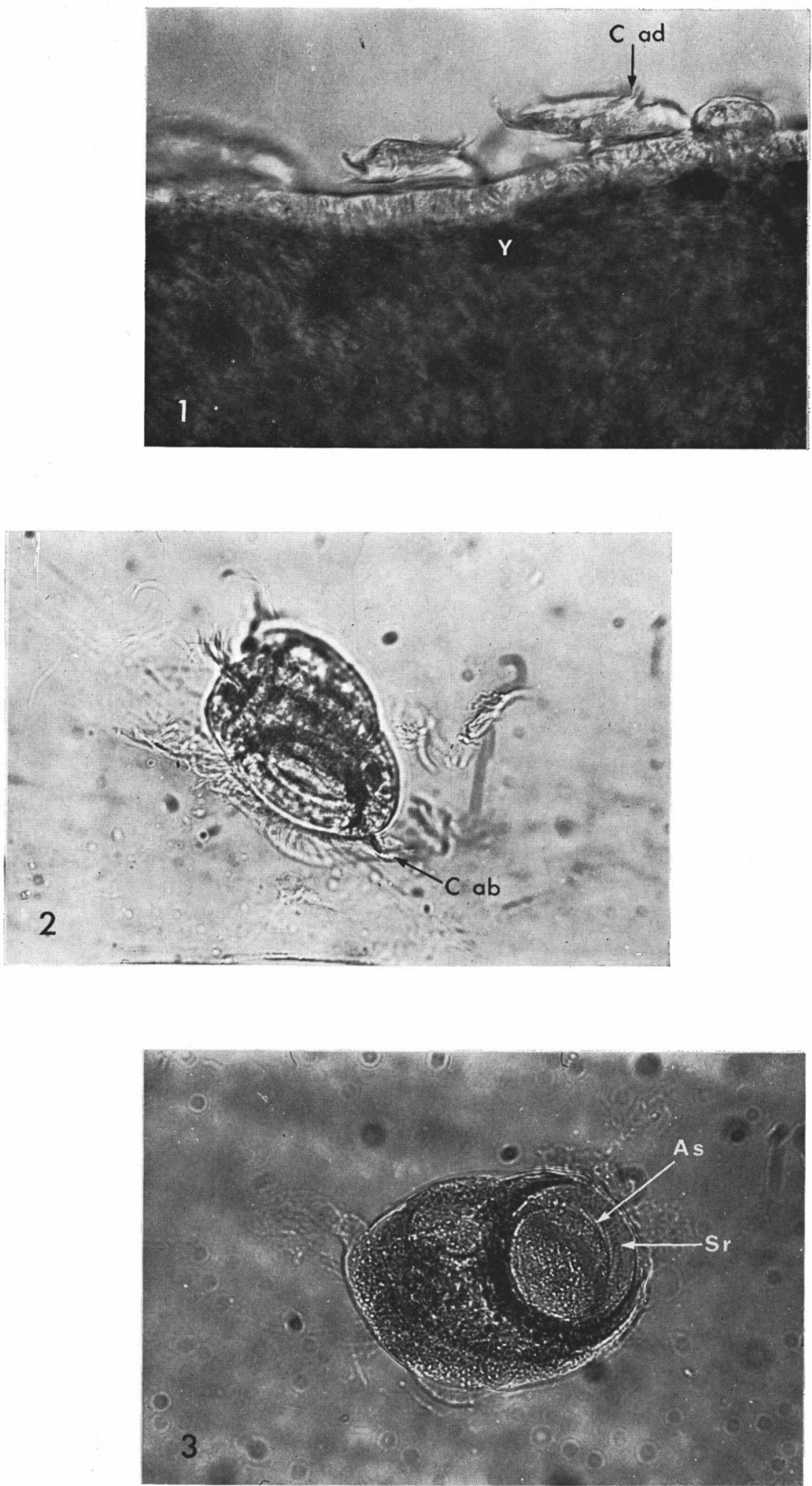

Fig. 1. - Urceolaria mitra. -1 : Fixées sur P. tenuis $(\times 280)$. y $=$ yeux de la Planaire. C. ad. = ciliature aborale; 2 : Individu détaché vu de $3 / 4(\times 440)$. C. ab. = ciliature aborale; 3 : Appareil adhésif $(\times 440)$. As $=$ Anneau squelettique. $\mathrm{Sr}=$ stries radiaires. 

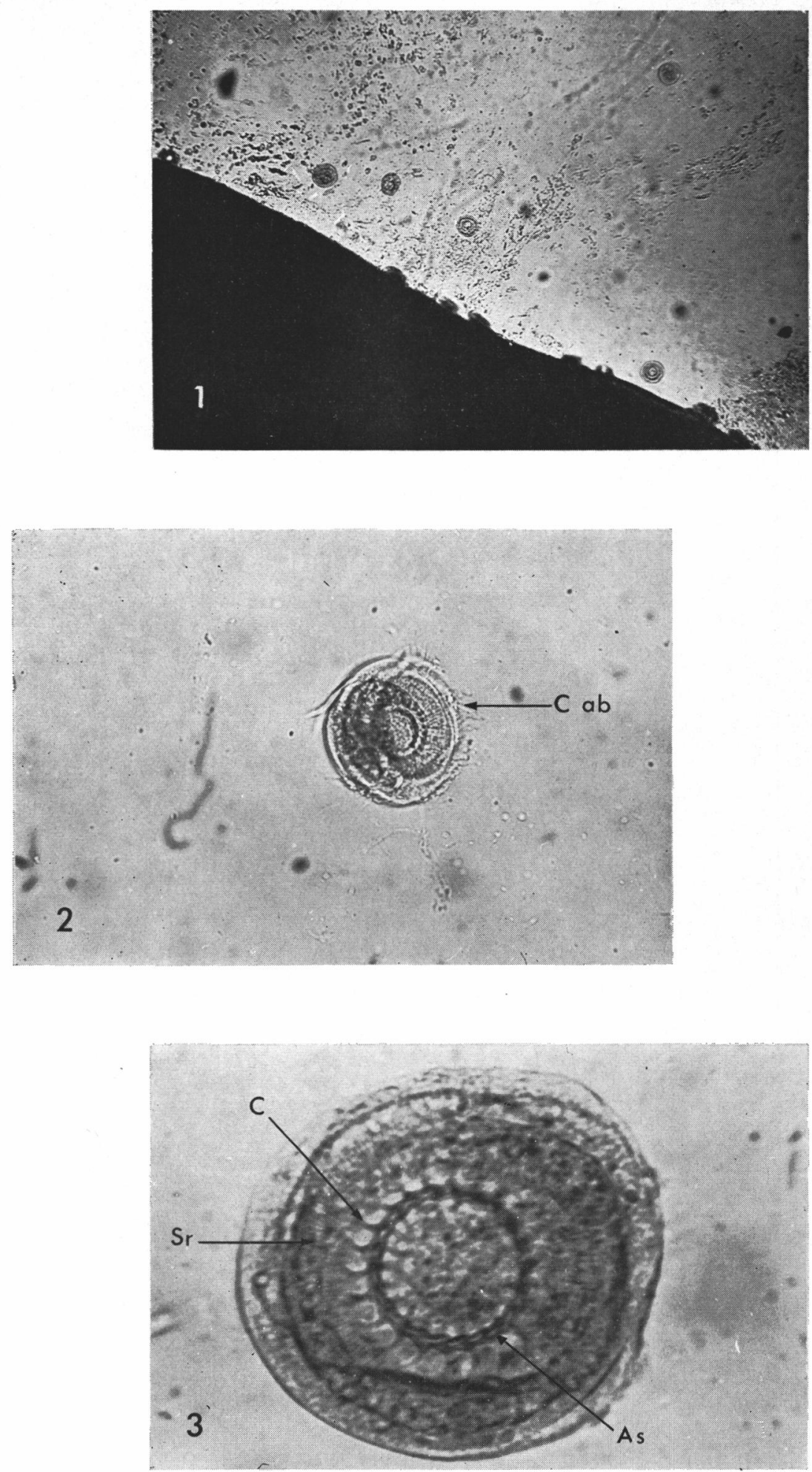

FIG. 2. - Trichodina steini. - 1 : Fixées ou en mouvement autour de P. nigra $(\times 50) ; 2$ : Individu détaché vu de $3 / 4(\times 440)$. C. ab = ciliature aborale; 3 : Détail de l'appareil adhésif ( $\times$ 990). As $=$ anneau squelettique. $\mathrm{C}=$ crochets. $\mathrm{Sr}=$ stries radiaires. 
Ces organismes sont étroitement inféodés à un mode de vie fixé et ne se trouvent à l'état planctonique que pour de brèves durées. Ils se nourrissent de particules en suspension dans l'eau (bactéries, diatomées, algues vertes et flagellés).

Nos connaissances sur la nature de l'association sont incertaines et les avis partagés. Reynoldson $(1950,1955)$ définit la nature de l'association sans la nommer, tandis que Penard (1922) parle de commensalisme et De Beauchamp (in Grassé, 1961) de parasitisme. Nous désignerons les membres de cette association sous le terme de commensaux, pris dans son sens étymologique : «qui mangent à la même table ».

Les Ciliés se rencontrent exclusivement sur le dos de l'hôte. Mais si tous les auteurs s'accordent sur ce point, les divergences apparaissent quant à la répartition dorsale des individus.

Reynoldson (1966) trouve $U$. mitra surtout abondante dans la région céphalique de l'hôte, zone qu'il définit comme particulièrement favorable à l'apport de nourriture par les courants ciliaires de la Planaire. Cette disposition est également celle que j'ai pu observer le plus fréquemment. Pour d'autres auteurs, par contre, $U$. mitra est uniformément répartie sur le dos de l'animal (Ball et Fernando 1968), ou montre une préférence très marquée pour la région caudale (Haider 1964, Ledfuss 1951). De telles contradictions peuvent s'expliquer selon Reynoldson (comm. pers.) par l'état d'activité différent des Planaires examinées et en particulier celui des cils antérieurs de l'animal.

Quant à $T$. steini, rencontrée par quelques auteurs sur $P$. nigra, sa répartition sur l'hôte peut être soit indifférente (Haider 1964), soit localisée à l'arrière de l'animal (Michel 1966, Hubault 1947). Ces deux possibilités se retrouvent sur les populations de la région lyonnaise.

En ce qui concerne la distribution des Ciliés entre les deux espèces jumelles de planaires, Reynoldson a remarqué que $U$. mitra était plus abondante sur $P$. tenuis que sur $P$. nigra. L'objet du présent travail est de compléter cette étude, en analysant les relations réciproques des deux Ciliés avec les deux Planaires.

\section{2. - COMPTAGE SUR DES INDIVIDUS PRELEVES DANS LA NATURE}

Ils ont porté sur des Planaires récoltées en de nombreuses stations de la région lyonnaise, mais également, dans la région parisienne et en Basse Provence dans un secteur déjà prospecté par Legier (1969) (fig. 3). 


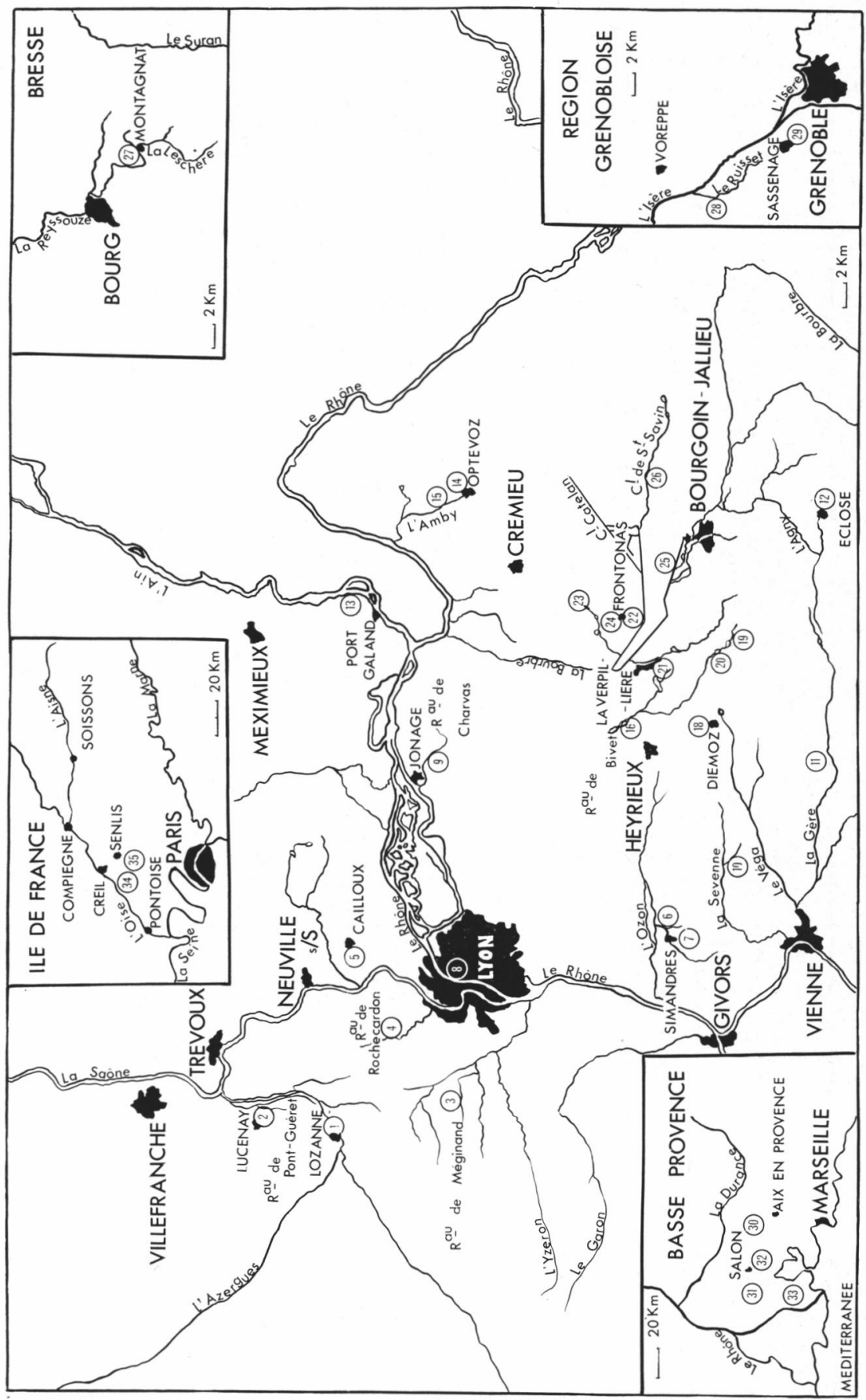

FIg. 3. - Origine des populations étudiées : carte de la situation des stations à $P$. nigra et à $P$. tenuis. Les numéros des stations correspondent à ceux du tableau I. 


\section{1. - Méthodes.}

\subsection{1. - RÉColte ET TRANSPORT DES ÉchaNTILlONS.}

Les Planaires ont été récoltées, dans toute la mesure du possible, pendant leur déplacement, alors qu'elles n'adhèrent que faiblement au support. Ce procédé évite les frottements sur l'animal. L'instrument, un pinceau fin, est passé à l'alcool, puis abondamment rincé à l'eau entre chaque prise. Dans le cas de populations hétérospécifiques de Protozoaires ou à plus forte raison de Planaires, ces dernières ont été conservées jusqu'au comptage, à raison d'une par pilulier. Enfin, les comptages ont été effectués dans des délais très brefs, une multiplication des Ciliés, motivée par on ne sait quel facteur, intervenant fréquemment au laboratoire quelques jours après la récolte.

Pour une plus grande homogénéité, les comptages ont porté sur des Planaires de taille voisine, correspondant à une taille adulte normale dans chaque station. Cette sélection répond aux travaux de Reynoldson (1951) qui observa une diminution numérique de la population d'épibiontes en deçà d'un certain gabarit de l'hôte.

\subsection{2. - Comptage des Protozoaires sur le dos de la Planaire.}

On utilise la loupe binoculaire $(\mathrm{G} \times 25$ et 40$)$ et un éclairage rasant.

Cette méthode, rapide - elle ne nécessite aucune préparation et fidèle - elle ne donne lieu à aucune manipulation, source possible d'erreur - présente cependant de nombreux inconvénients. Elle n'autorise pas le dénombrement rigoureux d'une population de Ciliés supérieure à 30 ou 40 individus par Planaire. Par ailleurs, elle ne permet pas de travailler sur des populations mixtes, lesquelles nécessitent à la fois une détermination et un comptage. Enfin, la mobilité de l'hôte rend parfois difficile l'observation. Son utilisation est donc forcément limitée.

\subsection{3. - Comptage des Protozoaires détachés.}

Reynoldson (1950) détachait les Ciliés de la Planaire en immergeant cette dernière $\mathbf{1 0}$ minutes dans une solution d'acide chromique à $0,02 \%$. Cependant à l'essai, la méthode s'est avérée peu satisfaisante dans le cas présent, car endommageant sévèrement la Planaire.

Une technique utilisant le vert de méthyle, mieux toléré par la Planaire, a été mise au point. Les animaux sont placés dans une lame creuse sur laquelle on dépose 3 à 4 gouttes de cette substance 
en solution à une concentration de 1 gramme par litre. La durée du traitement est variable en fonction de la densité de la population et de l'espèce de Protozoaire, T. steini se détachant moins facilement que $U$. mitra. En suivant l'opération à la loupe, on est à même de limiter cette durée pour obtenir une efficacité optimum, sans pour cela trop nuire à l'hôte (en cas d'agression violente de la part du liquide traitant, la Planaire émet en effet un mucus qui englue les Ciliés et rend l'opération de comptage malaisée). On retire ensuite la Planaire en la faisant glisser délicatement le long de la lame, où elle abandonne les dernières traces de solution et éventuellement les derniers Protozoaires. Le seul instrument utilisé à cet effet est une aiguille montée, très fine, ne retenant pas les épibiontes.

Afin de faciliter le dénombrement de populations très denses, les Planaires sont plongées dans 2 ou 3 bains successifs. Elles déposent alors sur chacune des lames une partie de leur colonie de Ciliés, que l'on peut compter aisément.

Le comptage s'effectue directement sur la lame creuse sans lamelle, en utilisant le microscope avec un grossissement de 20 à 32 , et en balayant systématiquement la préparation suivant des bandes transversales ou longitudinales. Ce faible grossissement ne permettant pas toujours l'identification de l'individu, il est souvent nécessaire de reprendre l'observation avec un objectif plus fort. On repère alors le champ à l'aide de la platine graduée.

Les comptages portent sur 30 Planaires par station. Lorsque $P$. nigra et $P$. tenuis coexistent, les résultats n'intéressent donc pas un nombre égal de Planaires de chaque espèce. En effet, la représentation des deux formes étant inégale, il eut fallu parfois récolter plus de 200 individus (à raison d'un animal par pilulier) pour obtenir 2 séries de 30.

- Valeur de la méthode. — La précision obtenue est très acceptable. Les comptages effectués sur des populations peu denses, en place sur le dos de la Planaire, puis détachées, le démontrent : pour une colonie de $\mathbf{4 0}$ Ciliés, il est rare de trouver une différence de plus de 2 ou 3 individus entre les deux méthodes. Après de multiples vérifications il est permis de penser que la marge d'erreur de la méthode ne dépasse généralement pas $5 \%$.

\section{2. - Résultats.}

\subsection{1. - Examen Des RÉSultats (Tableau I).}

Les moyennes des comptages varient de 0 à 236 Ciliés par Planaire.

- Stations à $P$. nigra : dans ces 7 stations, $T$. steini est seule représentée. 
Tableau I. - Nombre moyen de Protozoaires par Planaire sur des individus prélevés dans la nature.

Les valeurs aberrantes sont en italiques

Planaires Trichodina Urceolaria Planaires Trichodina Urceolaria observées steini mitra observées steini mitra

$\begin{array}{crrr}\text { Stations à } P \text {. nigra } & & & \\ \mathrm{n}^{\circ} \mathrm{I} & 30 & 16 & 0 \\ 2 & 30 & 125 & 0 \\ 3 & 30 & 214 & 0 \\ 6 & 8 & 0 & 0 \\ 16 & 10 & 33 & 0 \\ 25 & 30 & 17 & 0 \\ 30 & 17 & 0 & 0 \\ \text { moyenne x } & & 58 & 0\end{array}$

Station à $P$. tenuis

\begin{tabular}{|c|c|c|c|}
\hline & & & \\
\hline 8 & 30 & 0 & 39 \\
\hline 10 & 30 & 0 & 4 \\
\hline 11 & 30 & 0,7 & 84 \\
\hline 12 & 30 & 26 & 109 \\
\hline 14 & 30 & 0,9 & 34 \\
\hline 15 & 30 & 72 & 55 \\
\hline 18 & 30 & 62 & 47 \\
\hline 19 & 30 & 0 & 0,03 \\
\hline 20 & 30 & 0 & 21 \\
\hline 21 & 30 & 1 & 116 \\
\hline 23 & 4 & 7 & 32 \\
\hline 24 & 30 & 0,2 & 20 \\
\hline 27 & 30 & 2 & 105 \\
\hline 28 & 30 & 0 & 44 \\
\hline 31 & 15 & 13 & 236 \\
\hline 32 & 45 & 0 & 9 \\
\hline 33 (Jouine) & 30 & 103 & 2 \\
\hline enne $x$ & & 17 & 56 \\
\hline
\end{tabular}

\section{Stations mixtes}

$\mathrm{n}^{\circ} \quad 4$
5
7
9
13
22
26
29
34
35

2
27
21
18
7
12
1
19
24
1

$\begin{array}{rl}32 & 0 \\ 0 & 0,03 \\ 143 & 0,3 \\ 26 & 0,1 \\ 6 & 0 \\ 69 & 11 \\ 137 & 0 \\ 113 & 4 \\ 34 & 2 \\ 9 & 5\end{array}$

11
3
9
12
23
24
26
11
1
10

163 
— Stations à $P$. tenuis : les résultats sont ici moins catégoriques. $U$. mitra est dominante mais non exclusive. T. steini est parfois présente, mais en petit nombre, à l'exception du ruisseau du Moulin (station $\mathrm{n}^{\circ} 18$ ), de l'étang sur l'Amby (station $\mathrm{n}^{\circ}$ 15) et surtout du fossé affluent de la Jouine en Basse Provence (station $\mathrm{n}^{\circ} 33$ ), qui montrent des valeurs radicalement opposées. - Stations mixtes : les résultats confirment les précédentes observations. Notons au passage, l'intérêt de tels milieux dans lesquels les deux espèces de Protozoaires présentes peuvent véritablement choisir leur hôte.

\subsection{2. - Analyse des Résultats (Tableau II).}

Dans les stations à $P$. nigra, les résultats se passent d'analyse puisque seule une espèce de Ciliés est représentée. Le traitement statistique des données montre dans les stations à $P$. tenuis, un écart significatif entre les moyennes des dénombrements de $U$. mitra et de T. steini. Enfin, dans les stations mixtes, se dégage dans tous les cas une différence au moins significative, parfois même hautement significative, que ce soit entre le nombre de $T$. steini et d'U. mitra portés par chaque espèce de Planaire, ou entre le nombre de $T$. steini ou d'U. mitra portés sur les deux espèces de Planaires.

Tableau II. - Analyse de variance relative au tableau I (selon Lison 1958).

D. L. : degrés de liberté

V. : carré moyen ou variance

F. : coefficient de Snedecor - Les signes * et * représentent des valeurs respectivement significatives et hautement significatives.

\begin{tabular}{|c|c|c|c|c|c|}
\hline & Analyse & $\begin{array}{l}\text { Source de } \\
\text { variation }\end{array}$ & D. L. & V & $\mathbf{F}$ \\
\hline $\begin{array}{l}\text { Stations à } \\
P \text {. tenuis }\end{array}$ & $\begin{array}{r}T . s t \text { et } U . m \\
\text { sur } P \text {. tenuis }\end{array}$ & $\begin{array}{l}\text { espèce } \\
\text { erreur }\end{array}$ & $\begin{array}{r}1 \\
15\end{array}$ & $\begin{array}{r}13172 \\
2272\end{array}$ & $5,79^{\star}$ \\
\hline \multirow{4}{*}{$\begin{array}{l}\text { Stations } \\
\text { mixtes }\end{array}$} & $\begin{array}{l}T . \text { st et } U . m \\
\text { sur } P \text {. nigra }\end{array}$ & $\begin{array}{l}\text { espèce } \\
\text { erreur }\end{array}$ & $\begin{array}{r}1 \\
18\end{array}$ & $\begin{array}{r}14936 \\
1525\end{array}$ & $9,79^{\star *}$ \\
\hline & $\begin{array}{l}T . \text { st et } U . m \\
\text { sur } P \text {. tenuis }\end{array}$ & $\begin{array}{l}\text { espèce } \\
\text { erreur }\end{array}$ & $\begin{array}{r}1 \\
18\end{array}$ & $\begin{array}{l}9129 \\
1164\end{array}$ & 7,84 * \\
\hline & $\begin{array}{l}T . \text { st sur } \\
P . \text { nigra } \\
\text { et } P . \text { tenuis }\end{array}$ & $\begin{array}{l}\text { espèce } \\
\text { erreur }\end{array}$ & $\begin{array}{r}1 \\
18\end{array}$ & $\begin{array}{r}14434 \\
1526\end{array}$ & $9,46^{\star \star}$ \\
\hline & $\begin{array}{l}U . m \text { sur } \\
P . \text { nigra } \\
\text { et } P \text {. tenuis }\end{array}$ & $\begin{array}{l}\text { espèce } \\
\text { erreur }\end{array}$ & 18 & $\begin{array}{l}9529 \\
1164\end{array}$ & 8,19 * \\
\hline
\end{tabular}


Ainsi, sans être vraiment stricte, cette spécificité statistique est néanmoins l'expression évidente d'une préférence marquée de chacun des Ciliés vis-à-vis de l'une des deux Planaires.

Il a paru également intéressant de calculer les coefficients de corrélation r, en particulier dans les stations à Polycelis hétérospécifiques.

\begin{tabular}{lll}
\hline \multicolumn{1}{c}{ espèce intéressée } & \multicolumn{2}{c}{ variables } \\
\hline T. steini & P. n. et P. t. & $\mathrm{r}$ \\
$\begin{array}{l}\text { U. mitra } \\
\text { P. nigra }\end{array}$ & P. n. et P. t. & 0,56 \\
$P$. tenuis & T. st et U. m & 0,11 \\
\hline
\end{tabular}

Valeur critique de $\mathrm{r}$ pour le niveau de probabilité $5 \%=0,57$ (selon Dagnelie 1969).

L'analyse du tableau précédent comporte les 2 points suivants :

1) Relation entre le nombre de Protozoaires de la même espèce chez les deux espèces de Planaires.

La corrélation est positive et significative à peu de chose près chez $T$. steini. Autrement dit, cette espèce tend à être d'autant mieux représentée sur $P$. tenuis qu'elle est plus abondante sur $P$. nigra. Le nombre d'individus portés par $P$. tenuis varie donc en fonction directe de la taille de la population. Nous retrouvons là un phénomène déjà observé par Reynoldson (1950) à propos de la présence de $U$. mitra sur Dugesia lugubris. Si la population de Potozoaires possède une très forte densité, les individus montrent une attirance moins marquée pour leur hôte habituel et peuvent se reporter sur une autre espèce de Planaire. Ce phénomène est d'ailleurs bien connu comme facteur de contrôle des populations (Solomon 1949).

En ce qui concerne $U$. mitra, la valeur de $r$ est loin d'être significative. On peut donc penser que, à l'inverse de ce qui se passe pour T. steini, $P$. nigra ne reçoit pas l'excédent de population d'U. mitra porté par $P$. tenuis.

2) Relations entre le nombre de Protozoaires des deux espèces chez la même espèce de Planaire.

L'analyse montre l'absence certaine de corrélation, révélant ainsi pour une même population, une certaine indépendance des deux Ciliés l'un par rapport à l'autre. Elle s'insère, elle aussi, dans l'optique de Reynoldson (1955), qui, étudiant $U$. mitra sur $P$. tenuis, négligeait la présence éventuelle de $T$. steini sur l'hôte 
comme n'influençant pas l'autre espèce. Sans aller jusque là, faute de preuves plus solides, on peut cependant présumer de l'absence de compétition entre les deux espèces de Protozoaires.

\section{3. - Conclusion.}

Point n'est besoin d'insister sur le caractère d'affinité marquant les relations de $T$. steini à $P$. nigra et de $U$. mitra à $P$. tenuis : les chiffres sont assez éloquents. Le phénomène, s'il n'est pas expliqué - ce n'était d'ailleurs pas le but de ces comptages est ainsi mis en évidence, avec certitude.

On ne doit pas pour autant oublier la présence des 3 stations apparemment aberrantes - le fossé de la Jouine en étant l'exemple extrême - suffisamment exceptionnelles pour ne pas mettre en doute la valeur d'ensemble des résultats, mais assez surprenantes pour inciter à la réflexion. Comment se fait-il que $U$. mitra y soit moins bien représentée sur $P$. tenuis que $T$. steini, voire presque absente ? Quelle en est la cause ? Le milieu ? Un comportement particulier des Planaires ou des Protozoaires?

- Le milieu : les facteurs chimiques n'apparaissent pas importants à Noland (1925) qui décrit la grande tolérance des Ciliés à leur endroit. Par ailleurs, l'analyse chimique des eaux du ruisseau du Moulin (station $\mathrm{n}^{\circ} 18$ ) ne révèle aucune valeur remarquable.

- Les Planaires : elles possèdent une morphologie identique, et même si quelque particularité les différencie, il est peu probable que les Protozoaires y soient sensibles. La pigmentation ne semble pas avoir plus d'importance : en aucun cas elle n'a paru modifier le choix des Ciliés, confirmant ainsi une étude de Reynoldson (1950) sur U. mitra.

On peut évidemment imaginer l'intervention d'un phénomène attractif biochimique particulier. Mais ce domaine reste totalement inconnu.

- Les Protozoaires : des expériences de choix ont été réalisées afin d'étudier leur comportement en face de Planaires d'espèces et d'origines différentes.

\section{3. - EXPERIENCES DE CHOIX}

\section{1. - Principe.}

Il consiste à mettre en présence des Planaires infestées de Protozoaires avec d'autres Planaires dépourvues d'épibiontes. Les Ciliés ont donc la possibilité de changer de partenaires, et l'intensité du passage rend compte du niveau d'attraction. Sur un schéma voisin, Reynoldson (1956 a) a mené une série d'expériences concernant $U$. mitra et $P$. tenuis, avec des buts et des méthodes différents. 


\section{2. - Méthodes.}

\subsection{1. - Choix du Matériel animal.}

Cette phase est importante pour la réalisation de l'expérience dans des conditions optimales. Le choix s'est arrêté sur des Planaires portant des populations d'une cinquantaine de Protozoaires. Au-dessous de ce nombre, les passages sont trop peu nombreux; au-dessus, ils deviennent difficilement contrôlables.

Il est nécessaire aussi de pouvoir disposer d'un nombre important de Planaires, de multiples essais étant indispensables pour mettre au point les conditions d'expérience : on choisit donc seulement des stations permettant une récolte abondante.

Afin d'obtenir des résultats comparables, il est bon, également, que les échantillons proviennent de milieux écologiquement voisins. A ce sujet, les stations à faune mixte, dans lesquelles cohabitent $P$. nigra et $P$. tenuis, sont particulièrement intéressantes.

Enfin, les individus de la Jouine en Basse Provence (station $n^{\circ} 33$ ), pour lesquels les comptages de Ciliés ont donné des résultats surprenants, font l'objet d'une étude particulière.

\subsection{2. - Conduite DE L'EXPÉRIENCE.}

\section{- Elimination des Protozoaires.}

La technique d'élimination des Ciliés au vert de méthyle mise au point pour la présente étude procure rapidement un matériel varié et abondant. Un bain de 15 minutes dans une solution à 0,3 gramme par litre donne généralement de bons résultats sans léser par trop l'animal. Lorsque ce traitement ne suffit pas, on préfère renouveler l'opération 2 jours plus tard, plutôt que de prolonger l'immersion. La Planaire se rétablit rapidement et retrouve un comportement normal dans les 3 jours qui suivent. Cependant, un délai de 2 semaines est respecté avant d'expérimenter sur ces échantillons afin de permettre la régénération des tissus éventuellement lésés.

Une autre méthode a également été expérimentée sur certaines séries. Elle consiste à utiliser des individus nés et élevés au laboratoire, donc dépourvus de tout épibionte.

- Conditions expérimentales.

Quatre Planaires dépourvues de Protozoaires sont mises en présence de quatre Planaires infestées. L'utilisation de godets de petite taille $(5 \mathrm{~cm}$ de diamètre) favorise le contact entre les individus. Y contribue également la prise de nourriture (fragments de Lombrics ou Gammares blessés) qui, intervenant fréquemment mais par petites quantités, a pour effet, le temps du repas, de concentrer tous les animaux sur la proie. 
Le milieu - eau du robinet déchlorée par aération — est renouvelé une fois par semaine. Les conditions thermiques $\left(15^{\circ}\right)$ sont voisines des conditions d'origine.

Chacune des expériences de choix mettant les Ciliés en présence des deux espèces de Planaires, est menée parallèlement à un essai témoin rendant compte de l'intensité du passage entre Planaires de la même espèce.

La détermination des durées les plus favorables permettant d'obtenir des résultats clairs et facilement expoitables s'est faite par tâtonnement. Ces durées varient de 1 à 3 semaines, selon l'origine des individus et la nature de la population.

\section{- Contrôle de l'expérience.}

On suit l'évolution de jour en jour, au moyen de la loupe binoculaire. Pour distinguer les deux lots de Planaires, en particulier lorsque la pigmentation est identique, le marquage s'avère parfois indispensable. Une méthode a été mise au point : elle consiste à pratiquer une incision médiane longitudinale à l'extrémité postérieure de l'animal. Effectuéc au moyen d'un scapel affilé, la plaie cicatrise très rapidement faisant place à une queue bifide, visible environ 3 semaines. Divers essais ont montré que ce traitement n'avait pas d'influence décclable sur la population épizoïque.

En fin d'expérience, un comptage au vert de méthyle intervient. Il est bien entendu suivi d'une détermination méthodique de chaque Planaire traitée.

On assiste fréquemment, durant ces expériences, à une multiplication de la population de Protozoaires, principalement de $T$. steini. L'effectif total des individus de cette espèce peut quadrupler en 17 jours d'expérience, dépassant parfois 3000 ou 4000 individus par Planaire.

Ce phénomène peut être interprété comme une réaction des Ciliés aux conditions du milieu, soit directe sous l'influence de certains facteurs tels que la température ou l'alimentation, soit indirecte par suite d'une modification physiologique ou comportementale de l'hôte. Cette multiplication rend plus difficile le contrôle, mais ne paraît pas affecter la nature des relations entre les deux partenaires.

\subsection{3. - Présentation des RÉsultats.}

Les résultats sont ordonnés en 4 tableaux. Deux d'entre eux (Tableaux III et IV) ont trait au comportement de $U$. mitra d'une part et de $T$. steini d'autre part. Un troisième (Tableau V) rassemble les résultats obtenus à partir de populations mixtes de Ciliés, présentes sur $P$. tenuis. Cette dernière espèce est seule concernée par ce genre d'expérience, $P$. nigra n'ayant jamais été trouvée porteuse des deux espèces. Enfin, le dernier tableau (Ta- 
bleau VI) est uniquement consacré aux individus de la Jouine, partenaires d'une association Cilié-Planaire inhabituelle.

L'expression des résultats en pourcentage est rendue nécessaire par l'instabilité numérique des populations.

Les expériences correspondant aux conditions optimales ont été répétées.

\section{3. - Résultats.}

3.3.1. - EXAMEN des RÉSUltats.

- Populations monospécifiques de Ciliés.

$U$. mitra portée par $P$. tenuis (Tableau III) montre une spécificité très marquée pour cette même espèce : 40 à $45 \%$ des Ciliés changent de partenaire au sein de l'espèce $P$. tenuis. Au contraire, bien que le nombre de Ciliés sur la première puisse dépasser 600, le passage de $P$. tenuis à $P$. nigra est à peu près nul.

Tableau III. - Expérience de choix : passage d'U. mitra (au départ fixées sur $P$. tenuis) à $P$. nigra ou $P$. tenuis. Entre parenthèses, stations respectives d'origine des deux espèces de Planaires.

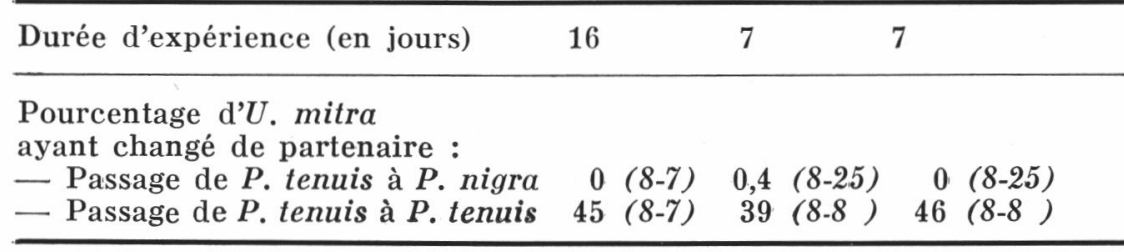

Pour T. steini présente sur $P$. nigra (Tableau IV), la différence est moins caractéristique mais bien marquée cependant et relativement régulière : on note en moyenne un rapport de 1 à 2 entre les échanges interspécifiques et intraspécifiques.

Aux nombreux échanges d'U. mitra entre les individus de $P$. tenuis, correspond donc une mobilité plus faible de T. steini.

TABleau IV. - Expérience de choix : passage de $T$. steini (au départ fixées sur $P$. nigra) à $P$. tenuis ou $P$. nigra. Entre parenthèses, stations respectives d'origine des deux espèces de Planaires.

\begin{tabular}{lllll}
\hline Durée d'expérience (en jours) & 7 & 21 & 17 & 17 \\
\hline $\begin{array}{l}\text { Pourcentage de } T \text {. steini ayant } \\
\text { changé de partenaire }\end{array}$ & & & & \\
- Passage de $P$. nigra à $P$. tenuis & $2(25-8)$ & $10(25-8)$ & $16(2-7)$ & $15(2-7)$ \\
- Passage de $P$. nigra à $P$. nigra & $8(25-25)$ & $33(25-25)$ & $27(2-7)$ & $24(2-7)$ \\
\hline
\end{tabular}

- Populations hétérospécifiques de Ciliés.

Ces expériences permettent de vérifier si le comportement d'individus des deux espèces cohabitant sur la même Planaire est iden- 
tique à celui de populations isolées. Cette identité se vérifie pour $U$. mitra (Tableau V) qui présente une affinité toujours très nette pour $P$. tenuis. Par contre, T. steini portée par $P$. tenuis dans la nature, conserve une préférence plus marquée pour cette espèce que pour $P$. nigra, pourtant partenaire habituel de l'association.

Tableau V. - Expérience de choix : passage de $T$. steini (au départ fixées sur le même hôte $P$. tenuis) à $P$. nigra et $P$. tenuis.

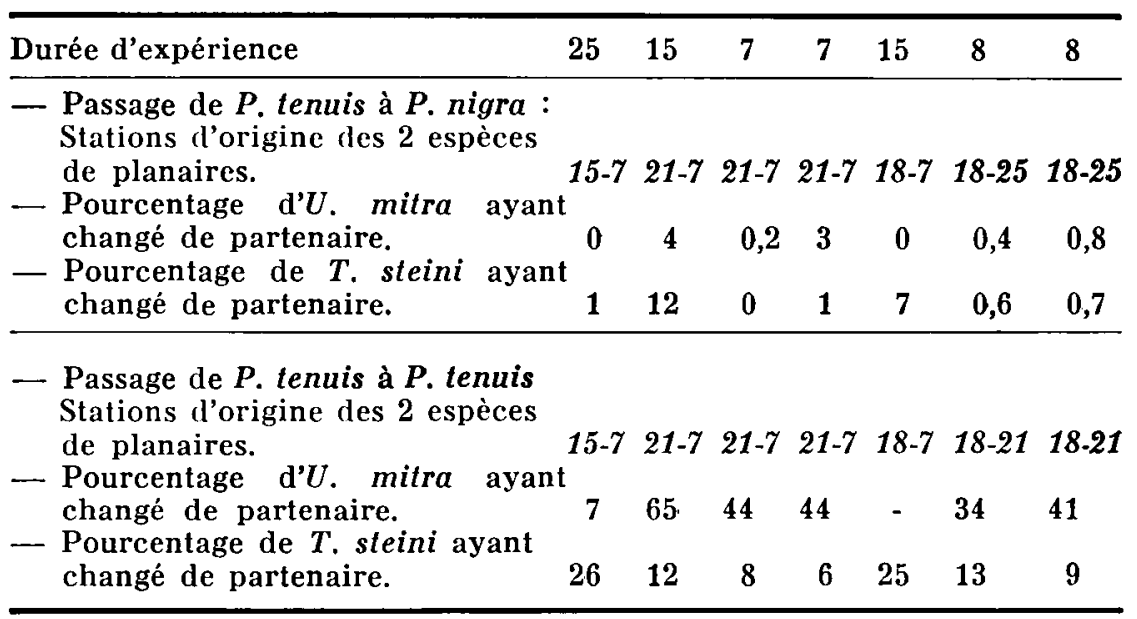

On trouve confirmation de ce phénomène avec les individus de la Jouine (Tableau VI). L'association $T$. steini - $P$. tenuis persiste même lorsque l'épibionte a le choix de son partenaire. On remarque qu'elle intéresse l'espèce $P$. tenuis entière, et non seulement les individus de la Jouine, avec toutefois une attirance plus nette pour ces derniers, ce qui paraît normal, les deux commensaux ayant une même origine.

Une contre-expérience complète ces données : $T$. steini provenant d'autres stations conserve son affinité pour $P$. nigra, même en présence de Planaires de la Jouine. 
TableaU VI. - Expérience de choix avec les Planaires de basse-Provence (fossé affluent de la Jouine) porteuses de populations particulières de Ciliés.

On distinguera ces $P$. tenuis de celles provenant de stations où l'association Cilié-Planaire est classique.

A) Passage de $T$. steini et $U$. mitra originellement fixées sur le même hôte, ( $P$. tenuis de la Jouine) à $P$. nigra et $P$. tenuis.

Durée d'expérience (en jours)

Pourcentage d'U. mitra ayant changé de partenaire :

1 - Passage de $P$. tenuis de la Jouine à $P$. nigra (Stat. $\mathrm{n}^{\circ} 25$ )

2 - Passage de $P$. tenuis de la Jouine à $P$. tenuis de la Jouine

3 - Passage de $P$. tenuis de la Jouine à $P$. tenuis (Stat. $\mathrm{n}^{\circ} 21$ )

Pourcentage de T. steini ayant changé de partenaire :

1 - Passage de $P$. tenuis de la Jouine à $P$. nigra (Stat. ${ }^{\circ}{ }^{25}$ )

2 - Passage de $P$. tenuis de la Jouine à $P$. tenuis de la Jouine

3 - Passage de $P$. tenuis de la Jouine à $P$. tenuis (Stat. $n^{\circ} 21$ )

B) Contre-expérience : passage de $T$. steini (originellement fixées sur P. nigra) à $P$. tenuis de la Jouine et à $P$. nigra.

Durée d'expérience (en jours)

Pourcentage de $T$. steini ayant changé de partenaire :

1 - Passage de $P$. nigra (Stat. $\mathrm{n}^{\circ}$ 2) à $P$. nigra (Stat. $\mathrm{n}^{\circ}$ 25)

2 - Passage de $P$. nigra (Stat. $\mathrm{n}^{\circ} 2$ ) à $P$. tenuis de la Jouine

3 - Passage de $P$. nigra (Stat. $\mathrm{n}^{\circ}$ 2) à $P$. tenuis (Stat. $\mathrm{n}^{\circ}$ 7)

\subsection{2. - Discussion.}

Au sujet de $\boldsymbol{U}$. mitra, ces expériences ne font que confirmer les précédents comptages qui, rappelons-le, révélaient l'absence de cette espèce sur $P$. nigra dans la nature.

Pour T. steini, tout n'est pas aussi simple. Ce Protozoaire se comporte différemment suivant qu'il est l'hôte habituel de $P$. nigra (Tableau IV) — c'est le cas le plus fréquent — ou de $P$. tenuis (Tableaux V et VIA). Si l'on en croit les comptages, ses préférences vont à $P$. nigra.

Lorsque, exceptionnellement, l'alliance $T$. steini-P. tenuis se réalise, qui, du Cilié ou de la Planaire en est responsable ? La réponse apparaît dans la contre-expérience réalisée avec les Planaires de la Jouine (Tableau VI B) : confrontées à une population de $T$. steini classique, c'est-à-dire portée par $P$. nigra, les $P$. tenuis de Basse Provence se révèlent beaucoup moins attractives que cette dernière espèce. Ce qui implique donc que, dans le fossé affluent 
de la Jouine, l'association apparemment aberrante est due à un comportement particulier du Protozoaire et non de la Planaire.

\section{4. - Conclusion.}

Dans les relations qui lient les 2 Ciliés aux Planaires, $U$. mitra apparait, sans conteste, comme l'épibionte spécifique de $\boldsymbol{P}$. tenuis.

Il faut, par contre, se montrer plus nuancé en ce qui concerne T. steini. Ce Cilié présente en fait deux formes, probablement deux races écologiques. L'une vit seule sur $P$. nigra : c'est de loin la plus fréquente. L'autre partage avec $U$. mitra le même hôte, $P$. tenuis, dominant même parfois très fortement (fossé affluent de la Jouine).

La situation mise en évidence dans la nature est stable. $T$. steini présente sur $P$. tenuis n'est pas un excédent de population rejeté d'ailleurs, mais une forme adaptée à son hôte particulier.

\section{4. - PERSPECTIVES ÉCOLOGIQUES}

Ces expériences ont mis en évidence une nouvelle distinction entre $P$. nigra et $P$. tenuis. Ceci tend à confirmer que ces deux espèces jumelles, trop souvent confondues, ne sont pas aussi proches qu'il paraît. Il n'est donc pas vain de vouloir en étudier séparément l'écologie.

En ce qui concerne la contribution de ce nouveau critère à la détermination des deux espèces jumelles, il apparaît que le comportement de $U$. mitra, qui n'est jamais équivoque, pourra être utilisé avec succès dans certains cas, en complément des caractères de reconnaissance habituellement employés. Ainsi, en présence d'une population mixte de $P$. nigra et $P$. tenuis, il sera possible de séparer les individus immatures de chaque espèce, soit par un simple examen de leur faune de $U$. mitra si celle-ci est. assez abondante, soit en les mettant en présence d'individus porteurs de ce Cilié et en observant les passages éventuels d'un hôte à l'autre.

Parmi les problèmes soulevés par cette étude, l'un des plus. intéressants est celui posé par la nature des phénomènes déterminant l'association de chacun des Ciliés avec son espèce affine de Planaire. Les relations entre les deux partenaires sont-elles. indirectes ou directes?

La première hypothèse en attribue la responsabilité aux facteurs du milieu : les deux partenaires généralement associés sont ceux qui montrent une affinité pour le même habitat. Cela supposedes différences écologiques entre les deux Planaires - ce qui est 
actuellement démontré (Reynoldson 1958, Lascombe 1971) — mais aussi entre les deux Ciliés - ce que nous ignorons.

Plus vraisemblable est l'hypothèse des relations directes : une attirance spécifique, motivée par on ne sait quel facteur, interviendrait, traduisant l'affinité des épibiontes pour leur espèce favorite, indépendamment des conditions d'habital. Ceci n'exclurait pas, bien entendu, une influence éventuelle du milieu sur les modalités de la fixation.

Se borner à mettre en évidence des faits ne suffit pas. Encore faut-il les comprendre, les expliquer. Au moment de clore cet article consacré à l'association Ciliés - Planaires, on entrevoit déjà une suite logique : l'étude des incidences écologiques de l'association sur l'épibionte et sur l'hôte. Nous saurons par là, si nous sommes en présence d'un vrai commensalisme.

\section{TRAVAUX GITES}

Ball (I.R.) and Fernando (C.H.). 1968. - On Urceolaria milta (Protozoa, Peritricha) epizoic on North American flatworms. Can. J. Zool., 46 : 981-985.

Beauchamp (P. de). 1961. - Classe des Turbellariés. In Grassé, traité de Zoologie, Paris, 4-1 : 35-212.

Dagnelie (P.). 1969. - Théorie et méthodes statistiques. Gembloux, 2 : 451 p.

HaIder $(\dot{G}$.). 1964. - Monographie der Familie Urceolariidae (Ciliata, Peritricha, Mobilia) mit besonderer Berücksichtigung der im suddentschen Raum vorkommenden Arten. Parasit. Schr., 17 :1-251.

Hubault (E.). 1947. - Etudes thermiques, chimiques et biologiques des eaux des lacs de l'Est de la France (Vosges, Jura, Alpes de Savoie). Ann. Ec. nat. Eaux et Forêts, 10-2 : 115.258.

KaHL (A.). 1935. - Urtiere oder Protozoa. I : Peritricha, Chonotricha. In DAHL Die Tierwelt Deutschlands, 30-4 : 651-886.

Lascombe (C.) et Pattee (E.). 1970. - L'affinité de deux Protozoaires Urceolariidés épizoïques pour les espèces de Planaires du genre Polycelis. Association Française de Limnologie, Super-Besse : 23-24.

- 1971. - Recherches écologiques et biogéographiques sur deux espèces jumelles de Planaires d'eau douce dans la région lyonnaise - Thèse Doctorat de Spécialité, Lyon, $116 \mathrm{p}$.

LEDFus (E.). 1951. - Bachplanarien aus der Umgebung von Erlangen (Ihre Verbreitung und der Befall mit Parasiten). Unveröffentlichte Staatsexamens-arbeit, $29 \mathrm{p}$.

LEGIER (P.). 1969. - Etude écologique des Planaires Triclades d'eau douce en Provence occidentale. Thèse Doctorat Spécialité, Marseille, $162 \mathrm{p}$.

Lender (T.). 1936. - Sur Polycelis nigra (Ehrbg.) et Polycelis tenuis (Ijima) Turbellariés Triclades. Archs zool. exp. gén., $78: 49.56$.

Lison (L.). 1958. - Statistique appliquée à la biologie expérimentale. Paris, 346 p.

Mrchel (S.). 1966. - Recherches sur la faune benthique du lac du Bour- . get. D.E.S. Lyon, $75 \mathrm{p}$.

NoLAND (L.E.). 1925. - Factors influencing the distribution of freswater ciliates. Ecology, 6 : 437-452. 
PAttee (E.). 1972. - Coefficients thermiques et écologie de quelques Planaires d'eau douce. V. La reproduction des espèces jumelles Polycelis nigra et Polycelis tenuis. Annls. Limnol., $8: 11-30$.

Penard (E.). 1922. - Etudes sur les Infusoires d'eau douce. Genève, 331 p. REYNOLDSON (T.B.). 1947. - Urceolarian epizoites of flatworms. Nature Lond., 160 : 265-266.

- 1948. - British species of Polycelis. (Plathyhelminthes). Nature Lond., 160 : 620-621.

- 1950. - Natural population fluctuations of Urceolaria mitra (Protozoa, Peritricha) epizoic on flatworms. J. Anim. Ecol., 19 : 106-118.

- 1951. - The dispersal of Urceolaria mitra (Peritricha) epizoic on flatworms. J. Anim. Ecol., $20: 123-131$.

- 1955. - Factors influencing population fluctuations of Urceolaria mitra (Peritricha) epizoic on freshwater triclads. J. Anim. Ecol. 24 : 57-83.

- 1956. - The population dynamics of host specificity in Urceolaria mitra (Peritricha) epizoic on freshwater triclads. J. Anim. Ecol., 25 : 127-143.

- 1958. - The quantitative ecology of lake-dwelling Triclads in northern Britain, Oikos, 9 : 94-138.

REYNOLdSON (T.B.). 1947. - Urceolarian epizoites of flatworms. Nature — dwelling Triclads towards a hypothesis. Adv. ecol. Res., $3: 1-71$.

Reynoldson (T.B.) et Bellamy (L.S.). 1970. - The establishment of interspecific competition in field populations, with an example of competition in action between Polycelis nigra (Müll) and $P$. tenuis (Ijima) (Turbellaria, Tricladida). Proc. Adv. Study Inst. Dynamics Numbers Popul. Oosterbeek 1970 : 282-297.

Solomon (M.E.). 1949. - The natural control of animal populations. J. Anim. Ecol., 18 : 1-35. 